\title{
Biembeddings of 2-rotational Steiner triple systems
}

\author{
M. J. Grannell \\ Department of Mathematics and Statistics \\ The Open University \\ Milton Keynes MK7 6AA, U.K. \\ m.j.grannell@open.ac.uk
}

\author{
J. Z. Schroeder \\ Department of Mathematical Sciences \\ George Mason University \\ Fairfax, VA 22030, U.S.A.
}

jzschroeder@gmail.com

Submitted: Sep 16, 2014; Accepted: May 7, 2015; Published: May 14, 2015

Mathematics Subject Classification: 05C10

\begin{abstract}
It is shown that for $v \equiv 1$ or $3(\bmod 6)$, every pair of Heffter difference sets modulo $v$ gives rise to a biembedding of two 2-rotational Steiner triple systems of order $2 v+1$ in a nonorientable surface.
\end{abstract}

Keywords: Topological embedding; Steiner triple system; Heffter difference set

\section{Introduction}

This paper is concerned with topologically embedding the complete graph $K_{n}$ in a surface such that all the faces are triangular. The surface may be orientable (a sphere with $g$ handles) or nonorientable (a sphere with $\gamma$ crosscaps) and the embedding should be cellular, meaning that the interior of each face should be homeomorphic to a disc. Euler's formula implies that a triangular embedding of $K_{n}$ is only possible when $n \equiv 0$ or 1 (mod $3)$ in the nonorientable case, and when $n \equiv 0,3,4$ or $7(\bmod 12)$ in the orientable case. Such embeddings provide minimum genus embeddings of $K_{n}$, and they arise naturally in the context of the Heawood map-colouring conjecture. It was established by Ringel and Youngs in the course of proving this conjecture that these necessary conditions are sufficient except in the nonorientable case $n=7$. Ringel's book [23] gives the details.

When $n \equiv 1$ or $3(\bmod 6)$ there is the possibility that a triangular embedding of $K_{n}$ is face 2-colourable, meaning that each face may be coloured with one of two colours in such a way that no two faces of the same colour share a common edge. In such cases, the triangular faces in each colour class form a Steiner triple system (formal definition below) of order $n$. We then say that the embedding is a biembedding of the two systems. The meaning of "biembedding" can be widened slightly by saying that system $S_{1}$ can be biembedded with system $S_{2}$ if there is a face 2-colourable triangulation of $K_{n}$ is which the systems formed by the two colour classes are isomorphic to $S_{1}$ and $S_{2}$. If the two systems 
formed by the colour classes are isomorphic to one another, then we speak of the embedding as a self-embedding. For general background material on topological embeddings, we refer the reader to [15] and [23], and for general background material on Steiner triple systems and related combinatorial designs, we refer the reader to [8] and [9].

After the pioneering work of Ringel and Youngs, many fundamental questions arise. These include determining a good lower bound on the number of minimum genus embeddings of $K_{n}$. For all sufficiently large $n, \mathrm{~V}$. P. Korzhik and H.-J. Voss used current graphs to establish a lower bound of the form $2^{\text {an }}[17,18,19,20]$. By using biembeddings of Steiner triple systems it has been shown that for certain infinite classes of $n$, a lower bound of the form $n^{a n^{2}}$ applies $[11,13]$. One may ask related questions about biembeddings themselves. In particular, does every pair of Steiner triple systems of order $n$ appear in a biembedding, and what can be said about self-embeddings? For some small values of $n$, these and associated questions were addressed by Bennett in his Ph.D. thesis [2], and some of his results appear in [3, 4, 5, 7]. The paper [12] gives all orientable biembeddings of the 80 nonisomorphic Steiner triple systems of order 15. Infinite classes of specific Steiner triple systems that are known to appear in biembeddings are somewhat rare. It is known that systems obtained from the Bose construction have self-embeddings in both orientable and nonorientable surfaces $[10,14]$. Although many instances of biembeddings of cyclic Steiner are known, it is not known whether or not every cyclic system appears in a biembedding. Our purpose in the current paper is to show that there is a large and well-structured class of Steiner triple systems, representatives of which appear in biembeddings. In order to describe this class we next give some formal definitions.

A partial triple system $\operatorname{PTS}(v, \lambda)$ is a pair $(V, \mathcal{B})$ where $V$ is a set of $v$ points and $\mathcal{B}$ is a collection of triples of points from $V$ such that each pair of points from $V$ occurs in at most $\lambda$ triples from $\mathcal{B}$. The triples in $\mathcal{B}$ are often called blocks. If each pair of points occurs exactly $\lambda$ times, then the system is called a $(\lambda$-fold $)$ triple system $\operatorname{TS}(v, \lambda)$. A triple system with $\lambda=1$ is called a Steiner triple system $\operatorname{STS}(v)$; such systems are known to exist if and only if $v \equiv 1$ or $3(\bmod 6)$ [16]. A permutation $\pi$ on the point set $V$ is said to be an automorphism of the system if it preserves $\mathcal{B}$. An automorphism is cyclic if it consists of a single cycle of length $v$, and $k$-rotational if it consists of one fixed point and $k$ cycles of equal lengths. Two systems, $(V, \mathcal{B})$ and $\left(V^{\prime}, \mathcal{B}^{\prime}\right)$, are said to be isomorphic if there is a bijection from $V$ to $V^{\prime}$ that takes $\mathcal{B}$ to $\mathcal{B}^{\prime}$.

A difference triple modulo $v$ is a triple from $\mathbb{Z}_{v}$ in which either (a) the three terms sum to $v$, or (b) the sum of two of the terms equals the third. Heffter's first difference problem is to construct a set of difference triples modulo $v=6 n+1$ that partition the set $\{1,2, \ldots, 3 n\}$. Such a set of difference triples will be denoted by $\operatorname{HDP}_{1}(n)$ and it can be used to construct an $\operatorname{STS}(v)(v=6 n+1)$ that has a cyclic automorphism of order $v$. If $\left\{\left\{a_{i}, b_{i}, c_{i}\right\}: 1 \leqslant i \leqslant n\right\}$ is an $\operatorname{HDP}_{1}(n)$, put $V=\mathbb{Z}_{v}$ and $\mathcal{B}=\left\{\left\{j, a_{i}+j, a_{i}+b_{i}+j\right\}\right.$ : $1 \leqslant i \leqslant n, j \in V\}$; then $(V, \mathcal{B})$ is an $\operatorname{STS}(v)$ with the automorphism $z \mapsto z+1$. An $\operatorname{HDP}_{1}(n)$ exists for each $n \geqslant 1$ [22]. Heffter's second difference problem is to construct a set of difference triples modulo $v=6 n+3$ that partition the set $\{1,2, \ldots, 3 n+1\} \backslash\{2 n+1\}$. Such a set of difference triples will be denoted by $\mathrm{HDP}_{2}(n)$ and it can be used to construct an $\operatorname{STS}(v)(v=6 n+3)$ that has a cyclic automorphism of order $v$. If $\left\{\left\{a_{i}, b_{i}, c_{i}\right\}\right.$ : 
$1 \leqslant i \leqslant n\}$ is an $\operatorname{HDP}_{2}(n)$, put $V=\mathbb{Z}_{v}$ and $\mathcal{B}=\left\{\left\{j, a_{i}+j, a_{i}+b_{i}+j\right\}: 1 \leqslant i \leqslant n\right.$, $j \in V\} \cup\{\{j, 2 n+1+j, 4 n+2+j\}: j \in V\}$; then $(V, \mathcal{B})$ is an $\operatorname{STS}(v)$ with the automorphism $z \mapsto z+1$. An $\operatorname{HDP}_{2}(n)$ exists for each $n \geqslant 2$ [22]. Observe that in both cases, $\mathrm{HDP}_{1}$ and $\mathrm{HDP}_{2}$, many distinct Steiner triple systems may result from re-writing $a_{i}, b_{i}, c_{i}$ in another order such as $a_{i}, c_{i}, b_{i}$.

There is a "doubling" construction for Steiner triple systems that takes a system of order $v$ and produces a system of order $2 v+1$. Suppose that $S=(V, \mathcal{B})$ is an $\operatorname{STS}(v)$. Take a copy of $K_{v+1}$ on a set $W$ of $v+1$ points, disjoint from $V$. Let $\mathcal{F}=\left\{F_{1}, F_{2}, \ldots, F_{v}\right\}$ be a 1 -factorization of this graph. For $i=1,2, \ldots, v$, place a distinct point $a_{i} \in V$ on each pair from $F_{i}$. Let $\mathcal{C}$ be the set of triples so formed. Then $(V \cup W, \mathcal{B} \cup \mathcal{C})$ is an $\operatorname{STS}(2 v+1)$.

The biembeddings that we construct are formed from two Heffter difference sets by applying the doubling construction with a suitable 1-factorization. In fact, we start with a "good" 1-factorization of $K_{v+1}$ (see the remark after the proof of Lemma 3) and then choose two appropriate $\operatorname{STS}(v)$ s arising from the given Heffter difference sets. The resulting STS $(2 v+1)$ s are 2-rotational. It follows that every pair of Heffter difference sets modulo $v$ gives rise to a biembedding of two 2-rotational STS $(2 v+1) \mathrm{s}$.

Our final comment in this section concerns notation. Given a set of points $V=$ $\left\{a_{1}, a_{2}, \ldots, a_{v}\right\}$, we take $\bar{V}$ to be a disjoint set of points $\left\{\overline{a_{1}}, \overline{a_{2}}, \ldots, \overline{a_{v}}\right\}$. We will often suppress brackets and commas when no confusion is likely, for example we may write a pair $\{a, b\}$ simply as $a b$.

\section{Constructing Embeddings}

Definition 1. Given a twofold partial triple system of order $v, \operatorname{PTS}(v, 2)=(V, \mathcal{B})$, we say that the ordered set of distinct points $a_{1} a_{2} \cdots a_{k}\left(a_{i} \in V\right.$ for $\left.i=1,2 \ldots, k\right)$ is a partial rotation at $a_{0} \in V$ if there is exactly one triple in $\mathcal{B}$ containing the pair $a_{0} a_{1}$, exactly one triple in $\mathcal{B}$ containing the pair $a_{0} a_{k}$, and the triples $a_{0} a_{i} a_{i+1}(i=1,2, \ldots, k-1)$ all lie in $\mathcal{B}$. We do not distinguish between the partial rotations $a_{1} a_{2} \cdots a_{k}$ and $a_{k} a_{k-1} \cdots a_{1}$. In a similar way we say that the cyclically ordered set of distinct points $a_{1} a_{2} \cdots a_{v-1}\left(a_{i} \in V\right.$ for $i=1,2 \ldots, v-1)$ is a full rotation at $a_{0} \in V$ if the triples $a_{0} a_{i} a_{i+1}(i=1,2, \ldots, v-2)$ and $a_{0} a_{v-1} a_{1}$ all lie in $\mathcal{B}$. We do not distinguish between the full rotations $a_{1} a_{2} \cdots a_{v-1}$ and $a_{v-1} a_{v-2} \cdots a_{1}$.

Given a triangular embedding of $K_{v}$ in a surface, the faces determine a triple system $\operatorname{TS}(v, 2)$. The cyclically ordered permutation of vertices around any given vertex $x$ will form a full rotation at $x$. Conversely, given a triple system $\operatorname{TS}(v, 2)$, if there is a full rotation at each point then this will determine an embedding of $K_{v}$ in a surface. The set of full rotations at each vertex in a triangular embedding is called a rotation scheme. Ringel's rule $\Delta^{*}$ [23] determines if the embedding corresponding to a rotation scheme is in an orientable or nonorientable surface: if the rotations at each vertex can be directed in such a way that, for each vertex $a$, whenever the rotation at $a$ contains the sequence $\ldots b c \ldots$, then the rotation at $b$ contains the sequence $\ldots c a \ldots$, then the embedding is in an orientable surface. If this cannot be done then the embedding is in a nonorientable surface. 
Definition 2. Suppose that $V=\{0,1, \ldots, v-1\}$ and $\bar{V}=\{\overline{0}, \overline{1}, \ldots, \overline{v-1}\}$ are sets of distinct points and that $\infty$ is a further distinct point. Given a triple $a b c$ of points from $W=V \cup \bar{V} \cup\{\infty\}$, we may derive further triples by applying the mappings

$$
\begin{gathered}
\phi_{j}: z \mapsto z+j, \quad \bar{z} \mapsto \overline{z+j}, \quad \infty \mapsto \infty \quad(z, j \in V, \text { arithmetic modulo } v), \\
\psi: z \mapsto \bar{z}, \quad \bar{z} \mapsto z, \quad \infty \mapsto \infty \quad(z \in V) .
\end{gathered}
$$

If $\mathcal{A}$ is a set of triples $\left\{a_{i} b_{i} c_{i}: \quad i=0,1, \ldots, k\right\}$ of points from $W$, then we will denote by $\mathcal{A}^{*}$ the set of derived triples obtained by applying the mappings $\phi_{j}(j \in V)$ and $\psi$.

Lemma 3. Suppose that $m \geqslant 3$, that $v=2 m+1$, and that $V=\{0,1, \ldots, v-1\}$. For $i \in V \backslash\{0\}$ let $i^{\prime}$ denote $v-i$. Put

$$
\mathcal{A}=\left\{\left\{0, \bar{i}, \overline{(i+1)^{\prime}}\right\}: i=0,1, \ldots, m-1\right\} \cup\{\{0, \bar{m}, \infty\}\} .
$$

Then the derived set of triples $\mathcal{A}^{*}$ forms a PTS $(2 v+1,2)$ on the point set $W=V \cup \bar{V} \cup\{\infty\}$, and the following form the entire set of partial rotations at 0 :

$$
\begin{aligned}
& 2 i+1, \overline{i+1}, \overline{(i+2)^{\prime}},(2 i+5)^{\prime} \quad(0 \leqslant i \leqslant m-3) \\
& 1^{\prime}, \overline{0}, \overline{1^{\prime}}, 3^{\prime} \\
& 2^{\prime}, \bar{m}, \infty, \overline{m^{\prime}}, \overline{m-1}, 4^{\prime} .
\end{aligned}
$$

(The partial rotation $1^{\prime}, \overline{0}, \overline{1^{\prime}}, 3^{\prime}$ can be obtained from the general form $2 i+1, \overline{i+1}, \overline{(i+2)^{\prime}}$, $(2 i+5)^{\prime}$ by taking $i=-1$.)

At the point $\infty$ there is a full rotation

$$
0, \bar{m}, 2 m, \overline{3 m}, 4 m, \ldots, \overline{m^{\prime}} \text {. }
$$

Proof. The derived set of triples $\mathcal{A}^{*}$ has cardinality $2 v(m+1)=v(v+1)$, and therefore these triples cover $3 v(v+1)$ pairs. These pairs comprise two copies of each pair of the form $\{i, \bar{j}\}$ for $i, j \in V$, two copies of each pair of the forms $\{i, \infty\}$ and $\{\bar{i}, \infty\}$ for $i \in V$, and one copy of each pair of the forms $\{i, j\}$ and $\{\bar{i}, \bar{j}\}$ for $i, j \in V$ with $i \neq j$. Hence $\mathcal{A}^{*}$ forms a $\operatorname{PTS}(2 v+1,2)$.

The $m$ triples of the form $\left\{0, \bar{i}, \overline{(i+1)^{\prime}}\right\}$ each generate two further triples containing the point 0 , namely $\left\{\overline{i^{\prime}}, 0,(2 i+1)^{\prime}\right\}$ and $\{\overline{i+1}, 2 i+1,0\}$, making a total of $3 m$ triples. Hence, in $\mathcal{A}^{*}$ there are triples $\{0,2 i+1, \overline{i+1}\},\left\{0, \overline{i+1}, \overline{(i+2)^{\prime}}\right\}$ and $\left\{0, \overline{(i+2)^{\prime}},(2 i+5)^{\prime}\right\}$ for $i=$ $0,1, \ldots, m-3$. Assuming for the moment that there are no other triples in $\mathcal{A}^{*}$ that contain the pairs $\{0,2 i+1\}$ and $\left\{0,(2 i+5)^{\prime}\right\}$, these $3 m-6$ triples give the partial rotations at 0 of the form $2 i+1, \overline{i+1}, \overline{(i+2)^{\prime}},(2 i+5)^{\prime}$ for $0 \leqslant i \leqslant m-3$. The remaining six triples from this source containing the point 0 are $\left\{0, \overline{0}, \overline{1^{\prime}}\right\},\left\{\overline{0}, 0,1^{\prime}\right\},\left\{\overline{1^{\prime}}, 0,3^{\prime}\right\},\left\{0, \overline{m-1}, \overline{m^{\prime}}\right\},\left\{\bar{m}, 2^{\prime}, 0\right\}$ and $\left\{\overline{m-1}, 4^{\prime}, 0\right\}$. In addition, there are two triples containing the pair $\{0, \infty\}$, these are $\{0, \bar{m}, \infty\}$ and $\left\{\overline{m^{\prime}}, 0, \infty\right\}$. These eight triples give two apparent partial rotations at 0 namely $1^{\prime}, \overline{0}, \overline{1^{\prime}}, 3^{\prime}$ and $2^{\prime}, \bar{m}, \infty, \overline{m^{\prime}}, \overline{m-1}, 4^{\prime}$. To confirm that all the sequences identified as potential partial rotations are indeed partial rotations, observe that there are no further triples of $\mathcal{A}^{*}$ that contain the point 0 . 
The triples containing the point $\infty$ are

$$
\{\{0, \bar{m}, \infty\}\},\{\bar{m}, 2 m, \infty\},\{2 m, \overline{3 m}, \infty\},\{\overline{3 m}, 4 m, \infty\}, \ldots,\left\{\overline{m^{\prime}}, 0, \infty\right\} .
$$

These give the full rotation at $\infty: 0, \bar{m}, 2 m, \overline{3 m}, 4 m, \ldots, \overline{m^{\prime}}$.

Note that the partial rotations at each point $j \in V$ may be obtained by applying the mapping $\phi_{j}$ to the partial rotations at 0 . The partial rotations at $\bar{j} \in \bar{V}$ may be obtained by applying the mapping $\psi$ to the partial rotations at $j$. The set $\mathcal{F}=\left\{F_{1}, F_{2}, \ldots, F_{v}\right\}$ where $F_{j}=\left\{\left\{\overline{i+j}, \overline{(i+1)^{\prime}+j}\right\}: i=0,1, \ldots, m-1\right\} \cup\{\{\overline{m+j}, \infty\}\}$ is a 1 -factorization of $K_{v+1}$ on the set $\bar{V} \cup\{\infty\}$.

Lemma 4. Given the set $\mathcal{A}$ from the previous lemma, form the set $\mathcal{B}$ by applying the mapping

$$
\chi: \quad z \mapsto 4^{-1} z, \quad \bar{z} \mapsto \overline{4^{-1} z}, \quad \infty \mapsto \infty \quad(z \in V \text {, arithmetic modulo } v) .
$$

Then the derived set of triples $\mathcal{B}^{*}$ forms a PTS $(2 v+1,2)$ and the following form the entire set of partial rotations at 0 :

$$
\begin{aligned}
& j, \overline{8^{-1}(4 j+1)}, \overline{\left[8^{-1}(4 j+3)\right]^{\prime}},(j+1)^{\prime} \quad(1 \leqslant j \leqslant m-1) \\
& m, \overline{4^{-1} m}, \infty, \overline{\left(4^{-1} m\right)^{\prime}}, \overline{4^{-1}(m-1)}, 1^{\prime} .
\end{aligned}
$$

At the point $\infty$ there is a full rotation

$$
0, \overline{4^{-1} m}, 2\left(4^{-1} m\right), \overline{3\left(4^{-1} m\right)}, 4\left(4^{-1} m\right), \ldots, \overline{\left(4^{-1} m\right)^{\prime}} .
$$

Proof. The derived set of triples $\mathcal{B}^{*}$ covers the same pairs with the same multiplicities as $\mathcal{A}^{*}$, so $\mathcal{B}^{*}$ forms a $\operatorname{PTS}(2 v+1,2)$ and has $v(v+1)$ triples. The full rotation at $\infty$ is immediate. Next observe that $\chi\left(2^{\prime}\right)=\left(2^{-1}\right)^{\prime}=(m+1)^{\prime}=m$, which also gives $\chi\left(4^{\prime}\right)=$ $2 m=1^{\prime}$. Hence we obtain the partial rotation $m, \overline{4^{-1} m}, \infty, \overline{\left(4^{-1} m\right)^{\prime}}, \overline{4^{-1}(m-1)}, 1^{\prime}$.

If $m$ is even, $\chi\left(1^{\prime}\right)=(m / 2)$ and $\chi\left(3^{\prime}\right)=(m / 2)-(m+1)=((m / 2)+1)^{\prime}$, and so we obtain the partial rotation $(m / 2), \overline{0}, \overline{(m / 2)},((m / 2)+1)^{\prime}$. If $m$ is odd, $\chi\left(3^{\prime}\right)=((m-1) / 2)$ and $\chi\left(1^{\prime}\right)=((m-1) / 2)+(m+1)=((m+1) / 2)^{\prime}$, and so we obtain the partial rotation $\left.((m-1) / 2), \overline{((m+1) / 2)^{\prime}}, \overline{0},((m+1) / 2)\right)^{\prime}$. Hence, applying $\chi$ to $1^{\prime}, \overline{0}, \overline{1^{\prime}}, 3^{\prime}$ gives the partial rotation $j, \overline{8^{-1}(4 j+1)}, \overline{\left[8^{-1}(4 j+3)\right]^{\prime}},(j+1)^{\prime}$ either for $j=(\mathrm{m} / 2)$ when $m$ is even, or for $j=((m-1) / 2)$ when $m$ is odd.

Note that as $i$ varies from 0 to $m-3,2 i+1$ and $(2 i+5)^{\prime}$ together take all values from 0 to $2 m$ apart from $0,2 m-3,2 m-2,2 m-1,2 m$. If we write $j=4^{-1}(2 i+1)$ and allow $i$ to vary from 0 to $m-3$ then $j$ and $(j+1)^{\prime}$ together take all values from 0 to $2 m$ apart from $0,1^{\prime}, 2^{-1}(m-1), m, 2^{-1} m$. If $m$ is even, $2^{-1} m=(m / 2)$ and $2^{-1}(m-1)=((m / 2)+1)^{\prime}$, while if $m$ is odd, $2^{-1} m=((m+1) / 2)^{\prime}$ and $2^{-1}(m-1)=((m-1) / 2)$. Hence, applying $\chi$ to the general form $2 i+1, \overline{i+1}, \overline{(i+2)^{\prime}},(2 i+5)^{\prime} \quad(0 \leqslant i \leqslant m-3)$ and writing $j=4^{-1}(2 i+1)$ gives the partial rotations $j, \overline{8^{-1}(4 j+1)}, \overline{\left[8^{-1}(4 j+3)\right]^{\prime}},(j+1)^{\prime} \quad(1 \leqslant j \leqslant m-1)$ apart from the case $j=(m / 2)$ for $m$ even and $j=((m-1)) / 2$ for $m$ odd. But this omission is covered by the previous paragraph. The result follows. 
As before, the partial rotations at each point $j, \bar{j}$ may be determined by applying the mappings $\phi_{j}$ and $\psi$. Working with $\mathcal{B}^{*}$ rather than $\mathcal{A}^{*}$ simplifies the statements and proofs of the following results. This is because the partial rotations at 0 provided by $\mathcal{B}^{*}$ have the simple form $j, \cdots,(j+1)^{\prime}$ for $0 \leqslant j \leqslant m-1$ and $m, \cdots, 1^{\prime}$. For future reference in the proof of Theorem 10, note how the sequence of numbers in the rotation at $\infty$ either consistently increments by $4^{-1} m$ or consistently decrements by the same amount, depending on the order in which it is written. Also note that the rotation at $\infty$ is invariant under the mapping $\psi$. We will show how the $\operatorname{PTS}(2 v+1,2)$ formed from $\mathcal{B}^{*}$ may be extended to a $\operatorname{TS}(2 v+1,2)$ using triples obtained from any two $\operatorname{HDP}_{1}(n) \mathrm{s}$ or any two $\operatorname{HDP}_{2}(n) \mathrm{s}$, according as $v=6 n+1$ or $6 n+3$. The extension will be such that the resulting $\operatorname{TS}(2 v+1,2)$ is decomposable into two block-disjoint $\operatorname{STS}(2 v+1) \mathrm{s}$, and there is a full rotation at every point.

Let $C$ be a weighted cycle on $m=3 n$ or $m=3 n+1$ vertices, with each edge having weight 1. Partition the vertices of $C$ into $n$ disjoint triples $T_{1}, T_{2}, \ldots, T_{n}$, together with a single vertex $\ell$ in the case when $m=3 n+1$. Next add the edges of each $T_{i}$ to $C$ and weight these 0 or 1 so that each $T_{i}$ has odd weight. In the case $m=3 n+1$, add a loop with weight 1 at the vertex $\ell$. Let $G$ denote the resulting weighted graph.

Lemma 5. The weighted graph $G$ has an Eulerian circuit $E$ that alternates edges of $C$ and edges of $G \backslash C$, and in which the sum of weights between the two occurrences of each vertex is odd.

Proof. Let $a$ be an arbitrary vertex of $G$. We will construct the circuit $E$ starting and ending at $a$. If a vertex $b$ is reached from $a$ in $E$ after traversing edges with odd weight sum, we record it as $b^{\prime}$, otherwise we record it as $b$. Thus the condition regarding the sum of weights between the two occurrences of the vertex $x$ is satisfied if and only if both $x$ and $x^{\prime}$ appear in our recording of the circuit $E$.

The proof is by induction. So consider first the case when $m=3$. Without loss of generality there are two possibilities. These are shown in Fig. 1 along with corresponding Eulerian circuits with the desired properties. Here, and subsequently, the underlined edges are those from the cycle $C$ which is represented by the outer circles $(a b c)$ in Fig. 1.
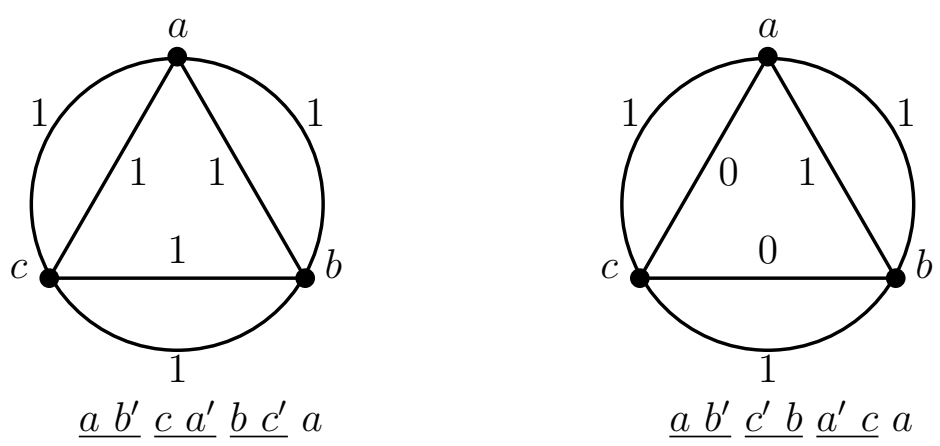

Figure 1: The case $m=3$.

Next we suppose that the problem is solved for $m=3 n$ vertices and we consider how to add three new vertices, say $x, y, z$, and a corresponding triangle of edges, and then form 
a new Eulerian circuit. There are several cases to consider.

(I) The first case is when the three new vertices are placed between three distinct pairs of existing consecutive vertices, say $\{a, b\},\{c, d\}$ and $\{e, f\}$ in the cycle $C$. Without loss of generality, we may assume that the original Eulerian circuit has one of the following four forms.
(i) $\underline{a b^{\prime}} \cdots \underline{c d^{\prime}} \cdots e \underline{e f^{\prime}} \cdots a$
(ii) $\underline{a b^{\prime}} \cdots \overline{c^{\prime} d} \cdots \overline{e f^{\prime}} \cdots a$
(iii) $\underline{a b^{\prime}} \cdots \underline{c d^{\prime}} \cdots \overline{e^{\prime} f} \cdots a$
(iv) $\overline{a b^{\prime}} \cdots \overline{c^{\prime} d} \cdots \overline{e^{\prime} f} \cdots a$

The additional triangle may have (a) three edges weighted 1 , or (b) one edge weighted 1 and the other two edges weighted 0 . These two options are shown in Fig. 2.

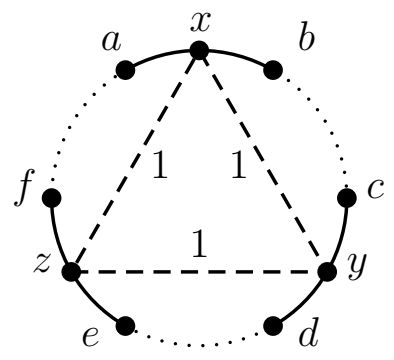

(a)

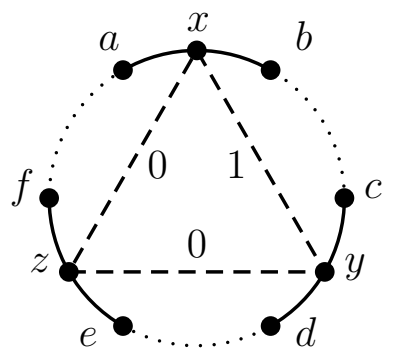

(b)

Figure 2: Adding $x y z$, case (I).

In the case (I)(a), the enlarged graph has one of the following four circuits.

(i) $a x^{\prime} y d^{\prime} \cdots \underline{e z^{\prime}} \underline{x b^{\prime}} \cdots c y^{\prime} z f^{\prime} \cdots a$

(ii) $\overline{a x^{\prime}} \frac{y c^{\prime}}{y} \cdots \overline{\frac{b^{\prime} x}{z^{\prime} e}} \cdots \overline{\frac{d y^{\prime}}{z f^{\prime}}} \cdots a$

(iii) $\underline{a x^{\prime}} \overline{y d^{\prime}} \cdots \overline{e^{\prime} z} \overline{y^{\prime} c} \cdots \overline{\frac{b^{\prime} x}{z^{\prime} f}} \cdots a$

(iv) $\underline{a x^{\prime}} \underline{z e^{\prime}} \cdots \underline{d y^{\prime}} \underline{\underline{x b^{\prime}}} \cdots \underline{c^{\prime} y} \overline{z^{\prime} f} \cdots a$

In the case $(\mathrm{I})(\mathrm{b})$, the enlarged graph has one of the following four circuits.

(i) $\underline{a} x^{\prime} y d^{\prime} \cdots \underline{e} z^{\prime} y^{\prime} c \cdots \underline{b^{\prime} x} z f^{\prime} \cdots a$

(ii) $\underline{a x^{\prime}} \underline{z^{\prime} e} \cdots d y^{\prime} \underline{x b^{\prime}} \cdots c^{\prime} y \overline{z f^{\prime}} \cdots a$

(iii) $a x^{\prime} y d^{\prime} \cdots \overline{e^{\prime} z} \underline{x b^{\prime}} \cdots \overline{c y^{\prime}} \overline{z^{\prime} f} \cdots a$

(iv) $\underline{a x^{\prime}} \underline{y c^{\prime}} \cdots \underline{b^{\prime} x} \underline{z e^{\prime}} \cdots \overline{d y^{\prime}} \overline{z^{\prime} f} \cdots a$

(II) The second case is when two of the new vertices are placed between one pair of existing consecutive vertices, say $\{a, b\}$, and the third new vertex is placed between a different pair of existing consecutive vertices, say $\{c, d\}$. Without loss of generality, we may assume that the original Eulerian circuit has one of the following two forms.

(i) $a b^{\prime} \cdots \underline{c d^{\prime}} \cdots a$

(ii) $\underline{a b^{\prime}} \cdots \overline{c^{\prime} d} \cdots a$

As before, the additional triangle may have three edges weighted 1 , or one edge weighted 1 and the other two edges weighted 0 . However, for this second possibility, without loss of generality, there are two possible placements for the triangle, giving three options altogether. These are shown in Fig. 3. 


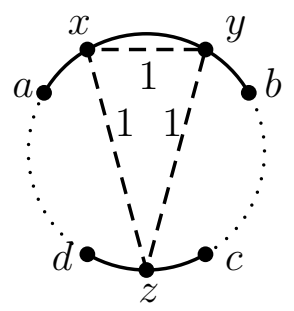

(a)

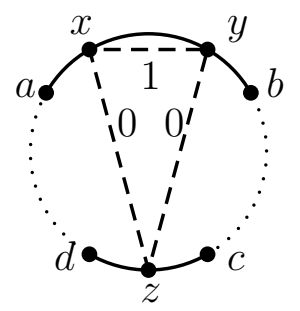

(b)

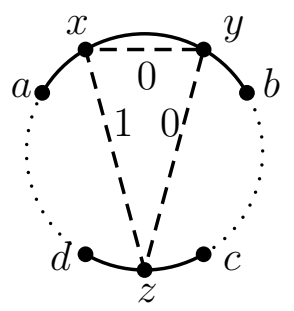

(c)

Figure 3: Adding $x y z$, case (II).

In the case (II)(a), the enlarged graph has one of the following two circuits.

(i) $a x^{\prime} y b^{\prime} \cdots \frac{c z^{\prime}}{a} \frac{x y^{\prime}}{z b^{\prime}} \cdots a$

(ii) $\underline{a x^{\prime}} \overline{y b^{\prime}} \cdots \frac{c^{\prime} z}{y^{\prime} x} \overline{z^{\prime} d} \cdots a$

In the case (II)(b), the enlarged graph has one of the following two circuits.

(i) $\underline{a} x^{\prime} \underline{y b^{\prime}} \cdots \underline{c z^{\prime}} \underline{y^{\prime} x} \underline{z d^{\prime}} \cdots a$

(ii) $\overline{a x^{\prime}} \frac{b^{\prime}}{y b^{\prime}} \cdots \overline{c^{\prime} z} \overline{x y^{\prime}} \frac{z^{\prime} d}{\cdots a} a$

In the case (II)(c), the enlarged graph has one of the following two circuits.

(i) $a x^{\prime} \underline{y^{\prime} x} \underline{z^{\prime} c} \cdots \frac{b^{\prime} y}{z} \underline{z d^{\prime}} \cdots a$

(ii) $\underline{a x^{\prime}} \underline{\underline{z c^{\prime}}} \cdots b^{\prime} y \overline{x y^{\prime}} \underline{z^{\prime} d} \cdots a$

(III) The third case is when all three of the new vertices are placed between one pair of existing consecutive vertices, say $\{a, b\}$. Without loss of generality, we may assume that the original Eulerian circuit has the following form.

$$
\underline{a b^{\prime}} \cdots a
$$

As before, the additional triangle may have three edges weighted 1 , or one edge weighted 1 and the other two edges weighted 0. However, for this second possibility, without loss of generality, there are two possible placements for the triangle, giving three options altogether. These are shown in Fig. 4.

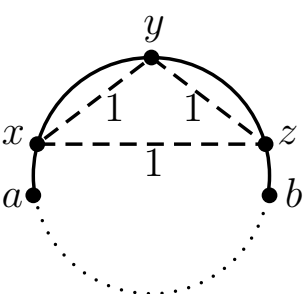

(a)

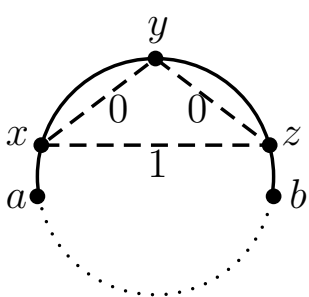

(b)

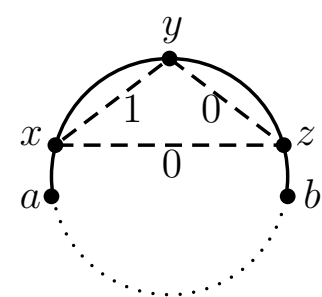

(c)

Figure 4: Adding $x y z$, case (III).

In the case (III)(a), the enlarged graph has the following circuit.

$$
\underline{a x^{\prime}} \underline{y z^{\prime}} \underline{x y^{\prime}} \underline{z b^{\prime}} \cdots a
$$

In the case (III)(b), the enlarged graph has the following circuit.

$$
\underline{a x^{\prime}} \underline{y^{\prime} x} \underline{z^{\prime} y} \underline{z b^{\prime}} \cdots a
$$

In the case (III)(c), the enlarged graph has the following circuit. 


$$
\underline{a x^{\prime}} \underline{y z^{\prime}} \underline{y^{\prime} x} \underline{z b^{\prime}} \cdots a
$$

Cases I, II and III cover all possibilities for adding a triangle to an existing circuit. It remains to consider how a loop may be added to deal with the case when the graph $G$ has $3 n+1$ vertices. So suppose a loop is to be added at a new vertex $\ell$ between the consecutive vertices $a$ and $b$ on the cycle $C$. Without loss of generality, we may assume that the original Eulerian circuit has the following form.

$$
\underline{a b^{\prime}} \cdots a
$$

Fig. 5 shows the placement of the loop.

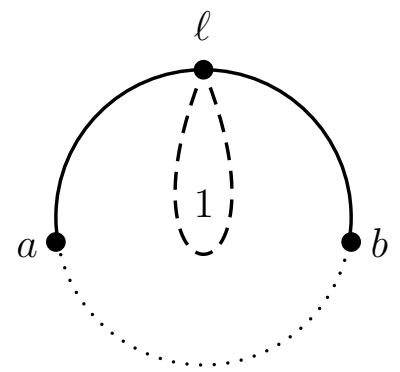

Figure 5: Adding a loop.

The enlarged graph has the following circuit.

$$
\underline{a \ell} \underline{\ell b^{\prime}} \cdots a
$$

This completes the inductive step and the result follows.

Observe that any Eulerian circuit $E$ with the properties specified in the preceding lemma has an additional property. If the cycle $C=\left(a_{1} a_{2} \cdots a_{m}\right)$, then $E$ may be written with the initial edge $a_{1} a_{2}^{\prime}$, that is $E=\left(\begin{array}{lll}a_{1} & a_{2}^{\prime} & \cdots\end{array}\right)$. In this form, and for each $i, E$ contains either the directed edge $a_{i} a_{i+1}^{\prime}$ or $\overline{a_{i+1}^{\prime}} a_{i}$, but neither $\underline{a_{i}^{\prime} a_{i+1}}$ nor $\underline{a_{i+1} a_{i}^{\prime}}$ (we take

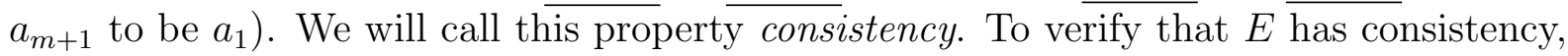
suppose the contrary. Then there is a smallest $i>1$ for which $E$ contains either $a_{i}^{\prime} a_{i+1}$ or $a_{i+1} a_{i}^{\prime}$. Since E also contains either $a_{i-1} a_{i}^{\prime}$ or $a_{i}^{\prime} a_{i-1}$, the vertex $a_{i}^{\prime}$ appears twice in $\bar{E}$, which is a contradiction.

By using Lemmas 4 and 5 and the observation about consistency, the main result may be established.

Theorem 6. Suppose that $v \equiv 1$ or $3(\bmod 6)$, and that $\mathcal{H}$ and $\overline{\mathcal{H}}$ are Heffter difference sets modulo $v$. Then there is a biembedding of two Steiner triple systems of order $2 v+1$, $\mathbf{S}$ and $\overline{\mathbf{S}}$, in which the two systems each have a 2-rotational automorphism, $\mathbf{S}$ contains a cyclic subsystem of order $v$ that arises from $\mathcal{H}$, and $\overline{\mathbf{S}}$ contains a cyclic subsystem of order $v$ that arises from $\overline{\mathcal{H}}$.

Proof. Consider first the case when $v=6 n+1, V=\mathbb{Z}_{v}$, and suppose that $\mathcal{H}=$ $\left\{T_{1}, T_{2}, \ldots, T_{n}\right\}$ and $\overline{\mathcal{H}}=\left\{\bar{T}_{1}, \bar{T}_{2}, \ldots, \bar{T}_{n}\right\}$ are $\operatorname{HDP}_{1}(n)$ s on $V$ and $\bar{V}$ respectively. 
To begin, we concentrate on $\mathcal{H}$. Let $C$ be the weighted cycle $(1,2, \ldots, m)(m=3 n)$ with each edge having weight 1 . If $T_{i}=\left\{a_{i}, b_{i}, c_{i}\right\}$, then add the edges $a_{i} b_{i}, b_{i} c_{i}, c_{i} a_{i}$ to $C$; if $a_{i}+b_{i}+c_{i}=v$ then weight all three edges with weight 1 , but if $a_{i}+b_{i}=c_{i}$ then weight the edge $a_{i} b_{i}$ with weight 1 and the other two edges with weight 0 . Do this for $i=1,2, \ldots, n$, and let $G$ denote the resulting weighted graph. By Lemma $5, G$ has an Eulerian circuit $E$ that alternates edges of $C$ and edges of $G \backslash C$, and in which the sum of the weights between the two occurrences of each vertex is odd. We will denote the two occurrences of a vertex $x$ in $E$ as $x$ or $x^{\prime}$ according as to whether or not the sum of weights between the vertex 1 and the vertex $x$ is even or odd. We will write $E$ with initial edge $\underline{12} 2^{\prime}$ and use underlining to indicate edges arising from $C$. We may also assume that $E$ has consistency.

If $T_{i}=\left\{a_{i}, b_{i}, c_{i}\right\}$ has $a_{i}+b_{i}+c_{i}=v$, then in $E$ the (undirected) edges arising from $T_{i}$ either appear as $a_{i}^{\prime} b_{i}, b_{i}^{\prime} c_{i}, c_{i}^{\prime} a_{i}$ or as $a_{i} b_{i}^{\prime}, b_{i} c_{i}^{\prime}, c_{i} a_{i}^{\prime}$. In the former case take $S_{i}$ to be any one of the three triples $\left\{0, a_{i}^{\prime}, b_{i}\right\},\left\{0, b_{i}^{\prime}, c_{i}\right\}$, or $\left\{0, c_{i}^{\prime}, a_{i}\right\}$; these are respectively the triples $\left\{0, b_{i}, b_{i}+c_{i}\right\},\left\{0, c_{i}, c_{i}+a_{i}\right\},\left\{0, a_{i}, a_{i}+b_{i}\right\}$ and they lie in a common orbit under $\phi_{1}$. Similarly in the latter case take $S_{i}$ to be any one of the three triples $\left\{0, a_{i}, a_{i}+c_{i}\right\}$, $\left\{0, b_{i}, b_{i}+a_{i}\right\},\left\{0, c_{i}, c_{i}+b_{i}\right\}$. If $T_{i}=\left\{a_{i}, b_{i}, c_{i}\right\}$ has $a_{i}+b_{i}=c_{i}$, then in $E$ the (undirected) edges arising from $T_{i}$ either appear as $a_{i}^{\prime} b_{i}, b_{i}^{\prime} c_{i}^{\prime}, c_{i} a_{i}$ or as $a_{i} b_{i}^{\prime}, b_{i} c_{i}, c_{i}^{\prime} a_{i}^{\prime}$. Here, in the former case take $S_{i}$ to be the triple $\left\{0, a_{i}, a_{i}+b_{i}\right\}$ and in the latter case take $S_{i}$ to be the triple $\left\{0, b_{i}, a_{i}+b_{i}\right\}$. Next put $\mathcal{S}=\left\{S_{1}, S_{2}, \ldots, S_{n}\right\}$, so that $\mathcal{S}$ forms a set of starters for a cyclic $\operatorname{STS}(v)$ on the point set $V$.

In an analogous way we deal with $\overline{\mathcal{H}}$, but on the point set $\bar{V}$. We obtain a weighted graph $\bar{G}$ with Eulerian circuit $\bar{E}$ and a set of starters $\overline{\mathcal{S}}$ for a cyclic $\operatorname{STS}(v)$ on $\bar{V}$.

By applying the mappings $\phi_{j}(j \in V)$ to $\mathcal{S}$ and to $\overline{\mathcal{S}}$ the triples of two cyclic $\operatorname{STS}(v) \mathrm{s}$ are obtained, one on the point set $V$ and the other on $\bar{V}$. These triples cover every pair of points from $V$ and every pair of points from $\bar{V}$ exactly once. Denote this set of triples by $\mathcal{C}^{*}$. The cardinality of $\mathcal{C}^{*}$ is $v(v-1) / 3$ and that of $\mathcal{B}^{*}$ is $v(v+1)$. Plainly $\mathcal{C}^{*} \cap \mathcal{B}^{*}=\emptyset$, so $\mathcal{D}=\mathcal{C}^{*} \cup \mathcal{B}^{*}$ is a set of $2 v(2 v+1) / 3$ triples that collectively cover every pair of points from $W=V \cup \bar{V} \cup\{\infty\}$ twice. Hence $(W, \mathcal{D})$ is a $\operatorname{TS}(2 v+1,2)$.

The triples from $\mathcal{D}$ may be partitioned into two sets $\mathcal{E}$ and $\mathcal{F}$. The former contains all triples from $\mathcal{D}$ of the forms $a b c$ and $a \bar{b} \bar{c}$ for $a, b, c \in V$, together with all triples of the form $\{j, \overline{m+j}, \infty\}$ for $j \in V$. The latter consists of the remaining triples from $\mathcal{D}$, namely those of the forms $\bar{a} \bar{b} \bar{c}$ and $\bar{a} b c$ for $a, b, c \in V$, together with all triples of the form $\{\bar{j}, m+j, \infty\}$ for $j \in V$. Then $\mathbf{S}=(W, \mathcal{E})$ and $\overline{\mathbf{S}}=(W, \mathcal{F})$ are $\operatorname{STS}(2 v+1)$ s whose union is the 2 -fold system $(W, \mathcal{D})$. The system $\mathbf{S}$ is a doubling of the cyclic system arising from $\mathcal{S}$ and consequently it contains a copy of this system. Similarly $\overline{\mathbf{S}}$ is a doubling of the cyclic system arising from $\overline{\mathcal{S}}$ and consequently it contains a copy of that system. Clearly $\phi_{1}$ is an automorphism of both $\mathbf{S}$ and $\overline{\mathbf{S}}$, proving that they are 2-rotational. Of course, the 2-fold system $(W, \mathcal{D})$ itself is 2-rotational.

It remains to prove that $(W, \mathcal{D})$ has a full rotation at every point of $W$. The full rotation at $\infty$ follows immediately from Lemma 4 . The rotation at 0 , and hence at all other points of $V$, is obtained from the Eulerian cycle $E$. An edge $a b$ in $E$ that comes from one of the $T_{i}$ 's has $a, b \in V$ and corresponds to a triple from $\mathcal{S}$, and hence to the 
triple $\{0, a, b\}$ of $\mathcal{D}$. Edges in $E$ that come from $C$ have the form $i(i+1)^{\prime}$; replace each of these by the corresponding partial rotation given by Lemma 4 , for example $\underline{12}$ is replaced by $1, \overline{8^{-1} 5}, \overline{\left[8^{-1} 7\right]^{\prime}}, 2^{\prime}$. These partial rotations correspond to the triples of $\mathcal{B}^{*}$ that contain the point 0 . Let $R$ denote the resulting list of points. Then $R$ is a cyclic permutation of all points of $W \backslash\{0\}$ and so forms a full rotation at 0 . By applying the mapping $\phi_{i}$ it follows that $(W, \mathcal{D})$ has a full rotation at every point $i \in V$. The rotation at $\overline{0}$ is obtained in a similar fashion using the Eulerian cycle $\bar{E}$ and the partial rotations formed by applying $\psi$ to the rotations given by Lemma 4 . It follows that $(W, \mathcal{D})$ has a full rotation at every point $i \in \bar{V}$. Consequently the 2 -fold triple system $(W, \mathcal{D})$ determines a triangular embedding of $K_{2 v+1}$. The embedding is face 2-colourable with the faces in each colour class corresponding to the Steiner triple systems $\mathbf{S}=(W, \mathcal{E})$ and $\overline{\mathbf{S}}=(W, \mathcal{F})$. Each of these systems has the 2-rotational automorphism $\phi_{1}$ and each contains a cyclic subsystem of order $v$ arising respectively from $\mathcal{H}$ and $\overline{\mathcal{H}}$.

Consider next the case when $v=6 n+3$ and $\mathcal{H}, \overline{\mathcal{H}}$ are $\operatorname{HDP}_{2}(n)$ s. The argument is very similar to the previous case but now $m=3 n+1$, the weighted graph $G$ has a loop with weight 1 at the point $2 n+1$, and there is an additional triple $S_{n+1}=\{0,2 n+1,4 n+2\}$. So now $\mathcal{S}=\left\{S_{1}, S_{2}, \ldots, S_{n+1}\right\}$ forms a set of starters for a cyclic $\operatorname{STS}(v)$ on the point set $V$ and the edge $\left\{2 n+1,(2 n+1)^{\prime}\right\}$ of $E$ corresponds to the triple $S_{n+1}$. The remaining details are as before.

Corollary 7. Suppose that $v \equiv 1$ or $3(\bmod 6)$ and that $\mathcal{H}$ is a Heffter difference set modulo $v$. Then there is a biembedding of two Steiner triple systems of order $2 v+1$ in which the two systems are isomorphic (and so this biembedding is a self-embedding), have a 2-rotational automorphism, and contain a cyclic subsystem of order $v$ that arises from $\mathcal{H}$.

Proof. The result follows from the theorem by taking $\overline{\mathcal{H}}$ to be $\psi(\mathcal{H})$, and using $\bar{G}=\psi(G)$, $\bar{E}=\psi(E), \overline{\mathcal{S}}=\psi(\mathcal{S})$.

Example 8. The case $\mathbf{v}=\mathbf{1 9}$.

Two Heffter difference sets $\operatorname{HDP}_{1}(3)$ are given on $V$ and $\bar{V}$ by $\mathcal{H}=\{\{1,3,4\},\{2,7,9\}$, $\{5,6,8\}\}$ and $\overline{\mathcal{H}}=\{\{\overline{1}, \overline{7}, \overline{8}\},\{\overline{2}, \overline{3}, \overline{5}\},\{\overline{4}, \overline{6}, \overline{9}\}\}$. Any Steiner triple system arising from $\mathcal{H}$ is nonisomorphic to any arising from $\overline{\mathcal{H}}[21]$. For $\mathcal{H}$ we have $1+3=4,2+7=9$ and $5+6+8=19$, so the weighted graph $G$ is as shown on the left of Fig. 6 . The edges of the cycle $C$ all have weight 1 and form the outer circle in the figure. The edges 13,27,56,58 and 68 all have weight 1, and the edges 14, 3429 and 79 have weight 0. Edges with weight 1 are shown with solid arcs and edges with weight 0 are shown with dotted arcs. The graph $\bar{G}$ corresponding to $\overline{\mathcal{H}}$ is obtained in an analogous manner to $G$ and is shown on the right of Fig. 6. 

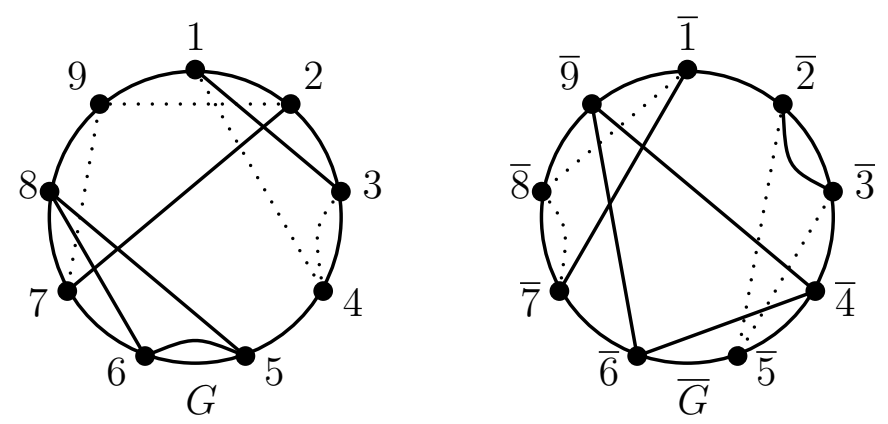

Figure 6: The weighted graphs $G$ and $\bar{G}$ for Example 8.

The partial rotations at 0 produced by Lemma 4 are as follows:

$1, \overline{3}, \overline{8^{\prime}}, 2^{\prime} ; \quad 2, \overline{13}, \overline{18^{\prime}}, 3^{\prime} ; \quad 3, \overline{4}, \overline{9^{\prime}}, 4^{\prime} ; \quad 4, \overline{14}, \overline{0}, 5^{\prime} ; \quad 5, \overline{5}, \overline{10^{\prime}}, 6^{\prime} ; \quad 6, \overline{15}, \overline{1^{\prime}}, 7^{\prime} ; 7, \overline{6}, \overline{11^{\prime}}, 8^{\prime} ;$ $8, \overline{16}, \overline{2}^{\prime}, 9^{\prime} ; \quad 9, \overline{7}, \infty, \overline{7^{\prime}}, \overline{2}, 1^{\prime}$.

By applying the method of Lemma 5 , the Eulerian circuits $E$ and $\bar{E}$ for $G$ and $\bar{G}$ are

$$
\begin{aligned}
& E=\underline{12^{\prime}} \underline{9^{\prime} 8} \underline{6^{\prime} 5} \underline{8^{\prime} 7} \underline{91^{\prime}} \underline{34^{\prime}} \underline{3^{\prime} 2} \underline{7^{\prime} 6} \underline{5^{\prime} 4}, \\
& \bar{E}=\underline{\overline{1} \overline{2^{\prime}}} \overline{3} \overline{4^{\prime}} \overline{6} \overline{7^{\prime}} \underline{8^{\prime} \overline{7}} \overline{1^{\prime}} \overline{9} \underline{6^{\prime}} \overline{5} \overline{2} \overline{3^{\prime}} \underline{\overline{5^{\prime}} \overline{4}} \overline{9^{\prime} \overline{8}} .
\end{aligned}
$$

Replacing the edges in $E$ that come from $C$ by the partial rotations and replacing $x^{\prime}$ by $v-x$ gives the rotation at 0 as

$$
\begin{aligned}
0: & 1, \overline{3}, \overline{11}, 17,10, \overline{17}, \overline{16}, 8,13, \overline{9}, \overline{5}, 5,11, \overline{8}, \overline{6}, 7,9, \overline{7}, \infty \\
& \quad \overline{12}, \overline{2}, 18,3, \overline{4}, \overline{10}, 15,16, \overline{1}, \overline{13}, 2,12, \overline{18}, \overline{15}, 6,14, \overline{0}, \overline{14}, 4
\end{aligned}
$$

In a similar way, the rotation at $\overline{0}$ is found to be

$$
\begin{gathered}
\overline{0}: \overline{1}, 3,11, \overline{17}, \overline{3}, 4,10, \overline{15}, \overline{6}, 15,18, \overline{12}, \overline{11}, 8,6, \overline{7}, \overline{18}, 2,12, \infty, \\
7, \overline{9}, \overline{13}, 9,5, \overline{5}, \overline{2}, 13,1, \overline{16}, \overline{14}, 0,14, \overline{4}, \overline{10}, 17,16, \overline{8}
\end{gathered}
$$

The rotations at $j$ and at $\bar{j}$ are obtained by applying $\phi_{j}$ respectively to the rotations at 0 and $\overline{0}$. The rotation at $\infty$ is

$$
\begin{array}{r}
\infty: \quad 0, \overline{7}, 14, \overline{2}, 9, \overline{16}, 4, \overline{11}, 18, \overline{6}, 13, \overline{1}, 8, \overline{15}, 3, \overline{10}, 17, \overline{5}, 12, \\
\quad \overline{0}, 7, \overline{14}, 2, \overline{9}, 16, \overline{4}, 11, \overline{18}, 6, \overline{13}, 1, \overline{8}, 15, \overline{3}, 10, \overline{17}, 5, \overline{12}
\end{array}
$$

These rotations define a biembedding of two (nonisomorphic) 2-rotational STS(39)s.

Example 9. The case $\mathbf{v}=\mathbf{1 5}$.

A Heffter difference set $\operatorname{HDP}_{2}(2)$ is given by $\mathcal{H}=\{\{1,3,4\},\{2,6,7\}\}$. Here we have $1+3=4$ and $2+6+7=15$, so the weighted graph $G$ is as shown in Fig. 7 . The edges of the cycle $C$ all have weight 1 and form the outer circle in the figure. The loop at vertex 5 and the edges 13,26,67 and 72 all have weight 1, and the edges 34 and 41 both have weight 0 . Edges with weight 1 are shown with solid arcs and edges with weight 0 are shown with dotted arcs. 


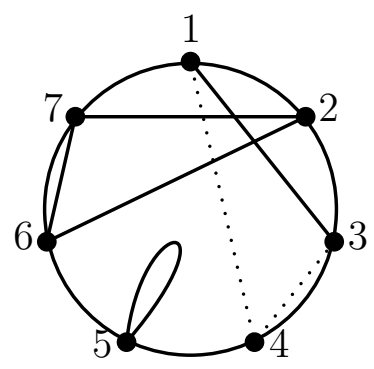

Figure 7: The weighted graph $G$ for Example 9 .

The partial rotations at 0 produced by Lemma 4 are as follows:

$1, \overline{5^{\prime}}, \overline{1}, 2^{\prime} ; \quad 2, \overline{3}, \overline{7^{\prime}}, 3^{\prime} ; \quad 3, \overline{4^{\prime}}, \overline{0}, 4^{\prime} ; \quad 4, \overline{4}, \overline{7}, 5^{\prime} ; \quad 5, \overline{3^{\prime}}, \overline{1^{\prime}}, 6^{\prime} ; \quad 6, \overline{5}, \overline{6}, 7^{\prime} ; 7, \overline{2^{\prime}}, \infty, \overline{2}, \overline{6^{\prime}}, 1^{\prime}$. By applying the method of Lemma 5 , the Eulerian circuit is

$$
E=\left(\underline{12^{\prime}} \underline{67^{\prime}} \underline{23^{\prime}} \underline{4^{\prime} 3} \underline{1^{\prime} 7} \underline{6^{\prime} 5} \underline{5^{\prime} 4}\right) .
$$

Replacing the edges from $C$ by the partial rotations and replacing $x^{\prime}$ by $v-x$ gives the rotation at 0 as

$$
0: 1, \overline{10}, \overline{1}, 13,6, \overline{5}, \overline{6}, 8,2, \overline{3}, \overline{8}, 12,11, \overline{0}, \overline{11}, 3,14, \overline{9}, \overline{2}, \infty, \overline{13}, 7,9, \overline{14}, \overline{12}, 5,10, \overline{7}, \overline{4}, 4
$$

The rotation at $j$ is obtained by applying $\phi_{j}$, and that at $\bar{j}$ by then applying $\psi$. The rotation at $\infty$ is

$$
\infty: 0, \overline{13}, 11, \overline{9}, 7, \overline{5}, 3, \overline{1}, 14, \overline{12}, 10, \overline{8}, 6, \overline{4}, 2, \overline{0}, 13, \overline{11}, 9, \overline{7}, 5, \overline{3}, 1, \overline{14}, 12, \overline{10}, 8, \overline{6}, 4, \overline{2}
$$

These rotations define a self-embedding of a 2-rotational STS(31).

To see that this embedding is in a nonorientable surface, suppose to the contrary, so that Ringel's rule $\Delta^{*}$ applies. If the rotation at 0 is taken in the order shown then $\Delta^{*}$ implies that the rotation at 1 must be in the order $1: \ldots, \overline{10}, 0, \ldots$ But the rotation at 1 is given by:

$$
\text { 1: } 2, \overline{11}, \overline{2}, 14,7, \overline{6}, \overline{7}, 9,3, \overline{4}, \overline{9}, 13,12, \overline{1}, \overline{12}, 4,0, \overline{10}, \overline{3}, \infty, \overline{14}, 8,10, \overline{0}, \overline{13}, 6,11, \overline{8}, \overline{5}, 5
$$

so the order of this must be reversed. But then $\Delta^{*}$ gives $\infty: \ldots, \overline{3}, 1, \ldots$, so the rotation at $\infty$ is in the order shown above. On the other hand, applying $\Delta^{*}$ directly to the rotation at 0 gives $\infty: \ldots, \overline{13}, 0, \ldots$, which is in the reverse order to that shown above. Hence the rotations at 0,1 and $\infty$ cannot be ordered to satisfy $\Delta^{*}$, and so the embedding is in a nonorientable surface.

The example above is indicative of the general case. We next prove that these embeddings are always in a nonorientable surface.

Theorem 10. Any embedding produced by the construction of Theorem 6 is in a nonorientable surface. 
Proof. Notation is taken from the earlier results. We first show that at least one difference triple in a Heffter difference set must be of the form $a+b=c$. Consider the sum of all the elements of all the difference triples. If $v=6 n+1$ this sum is $\sum_{i=1}^{3 n} i=$ $3 n(3 n+1) / 2$, which is coprime with $v$, while if $v=6 n+3$ this sum is $\left(\sum_{i=1}^{3 n+1} i\right)-(2 n+1)=$ $(3 n+1)(3 n+2) / 2-v / 3$, which is also coprime with $v$. Hence in either case, not all triples in a Heffter difference set can sum to $v$. The consequence for the graph $G$ is that at least one edge is weighted 0 , and therefore at least one edge of $E$ has the form $a b$ (both points unprimed) or $a^{\prime} b^{\prime}$ (both points primed). If $E$ is written with initial edge $\underline{12} 2^{\prime}$, then there must be a value $i(1 \leqslant i \leqslant m-1)$ for which $E$ has the directed edges $i(i+1)^{\prime}$ and $(i+2)^{\prime}(i+1)$ (if $i=m-1$, then $i+2$ is taken to be 1 ). It follows that the rotation at 0 has the form

$$
0: \ldots, \overline{\left[8^{-1}(4 i+3)\right]^{\prime}},(i+1)^{\prime}, \ldots, \overline{8^{-1}(4 i+5)},(i+1), \ldots
$$

and this rotation also contains either

(a) $\ldots, \overline{4^{-1} m}, \infty, \overline{\left(4^{-1} m\right)^{\prime}}, \ldots$ (in that order), or

(b) its reverse $\ldots, \overline{\left(4^{-1} m\right)^{\prime}}, \infty, \overline{4^{-1} m}, \ldots$

(If $i=m-1$ then $8^{-1}(4 i+5)=8^{-1}(4 m+1)=8^{-1}(2 m)=4^{-1} m$, so case (b) must then apply.)

Now suppose that rule $\Delta^{*}$ applies, then we have $i+1: \ldots, 0, \overline{8^{-1}(4 i+5)}, \ldots$ However, the rotation at $i+1$ is

$$
i+1: \ldots, \overline{\left[8^{-1}(4 i+3)\right]^{\prime}+(i+1)}, 0, \ldots, \overline{8^{-1}(4 i+5)+(i+1)}, 2(i+1), \ldots
$$

and $\left[8^{-1}(4 i+3)\right]^{\prime}+(i+1)=8^{-1}(4 i+5)$, so the order shown for this rotation must be reversed. The reversed rotation then contains in case (a)

$$
\ldots, \overline{\left(4^{-1} m\right)^{\prime}+(i+1)}, \infty, \overline{4^{-1} m+(i+1)}, \ldots,
$$

but in case (b)

$$
\ldots, \overline{4^{-1} m+(i+1)}, \infty, \overline{\left(4^{-1} m\right)^{\prime}+(i+1)}, \ldots
$$

Hence, by $\Delta^{*}$, in case (a) we have the rotation

$$
\infty: \ldots, \overline{4^{-1} m+(i+1)},(i+1), \ldots,
$$

while in case (b) we have

$$
\infty: \ldots, \overline{\left(4^{-1} m\right)^{\prime}+(i+1)},(i+1), \ldots
$$

But, applying $\Delta^{*}$ to the rotation at 0 gives, in case $(\mathrm{a}), \infty: \ldots, \overline{\left(4^{-1} m\right)^{\prime}}, 0, \ldots$ and, in case (b), $\infty: \ldots, \overline{4^{-1} m}, 0, \ldots$ In either case, there is a contradiction because the rotation at $\infty$ either consistently increments by $4^{-1} \mathrm{~m}$ or consistently decrements by the same amount, depending on the order in which it is written. Hence the rotations at $0, i+1$ and $\infty$ cannot be ordered to satisfy $\Delta^{*}$, and so the embedding is in a nonorientable surface. 


\section{Concluding remark}

The number of biembeddings of $\operatorname{STS}(2 v+1)$ s arising from the construction presented here is clearly related to the number of Heffter difference sets modulo $v$. Although the precise number of these is not known for general $v$, an estimate may be obtained from the number of Skolem sequences of various types, because each such sequence generates a Heffter difference set. There are four types of Skolem sequences: pure, hooked, split, and split-hooked. Abrham [1] showed that there are at least $2^{\left\lfloor\frac{n}{3}\right\rfloor}$ distinct pure Skolem sequences of order $n \equiv 0$ or $1(\bmod 4)$, and that the same bound applies to split Skolem sequences of order $n \equiv 0$ or $3(\bmod 4)$. It was shown in [2] (see also [6]) that the same bound applies for all sufficiently large $n$ to hooked and split-hooked Skolem sequences for (respectively) $n \equiv 2$ or $3(\bmod 4)$, and $n \equiv 1$ or $2(\bmod 4)$. A Skolem sequence (of an appropriate type) of order $n$ may be used to construct a Heffter difference set $\operatorname{HDP}_{1}(n)$ or $\operatorname{HDP}_{2}(n)$. However, computational evidence suggests that the estimate $2^{\left\lfloor\frac{n}{3}\right\rfloor}$ is very conservative.

\section{Acknowledgement}

The authors thank the anonymous referee whose suggestion enabled us to make a substantial improvement to our main result (Theorem 6).

\section{References}

[1] J. Abrham. Exponential bounds for the number of Skolem and extremal Langford sequences. Ars Combin., 22:187-198, 1986.

[2] G. K. Bennett. Topological embeddings of Steiner triple systems and associated problems in design theory. Ph.D. thesis, The Open University, 2004.

[3] G. K. Bennett, M. J. Grannell, and T. S. Griggs. Cyclic bi-embeddings of Steiner triple systems on 31 points. Glasg. Math. J., 43(1):145-151, 2001.

[4] G. K. Bennett, M. J. Grannell, and T. S. Griggs. Cyclic bi-embeddings of Steiner triple systems on $12 s+7$ points. J. Combin. Des., 10(2):92-110, 2002.

[5] G. K. Bennett, M. J. Grannell, and T. S. Griggs. Non-orientable biembeddings of Steiner triple systems of order 15. Acta Math. Univ. Comenian. (N.S.), 73(1):101106, 2004.

[6] G. K. Bennett, M. J. Grannell, and T. S. Griggs. Exponential lower bounds for the numbers of Skolem-type sequences. Ars Combin., 73:101-106, 2004.

[7] G. K. Bennett, M. J. Grannell, and T. S. Griggs, Orientable self-embeddings of Steiner triple systems of order 15. Acta Math. Univ. Comenian. (N.S.), 75(2):163$172,2006$.

[8] C. J. Colbourn and J. H. Dinitz (eds.). The Handbook of Combinatorial Designs (second edition). CRC Press, Boca Raton, 2007. 
[9] C. J. Colbourn and A. Rosa. Triple Systems. Oxford University Press, New York, 1999.

[10] P. M. Ducrocq and F. Sterboul. On G-triple systems. Publications du Laboratoire de Calcul de l'Université des Sciences et Techniques de Lille, 103, 18pp, 1978.

[11] M. J. Grannell and T. S. Griggs. A lower bound for the number of triangular embeddings of some complete graphs and complete tripartite graphs. J. Combin. Theory, Ser. B, 98(4):637-650, 2008.

[12] M. J. Grannell, T. S. Griggs, M. Knor, and A. R. W. Thrower. A census of the orientable biembeddings of Steiner triple systems of order 15. Australas. J. Combin., 42:253-259, 2008.

[13] M. J. Grannell and M. Knor. A lower bound for the number of orientable triangular embeddings of some complete graphs. J. Combin. Theory Ser B, 100(2):216-225, 2010.

[14] M. J. Grannell, T. S. Griggs, and J. Širáň. Surface embeddings of Steiner triple systems. J. Combin. Des., 6(5):325-336, 1998.

[15] J. L. Gross and T. W. Tucker. Topological Graph Theory. John Wiley, New York, 1987.

[16] T. P. Kirkman. On a problem in combinations. Cambridge and Dublin Math. J., 2:191-204, 1847.

[17] V. P. Korzhik. Exponentially many nonisomorphic orientable triangular embeddings of $K_{12 s}$. Discrete Math., 308(7):1046-1071, 2008.

[18] V. P. Korzhik and H.-J. Voss. On the number of nonisomorphic orientable regular embeddings of complete graphs. J. Combin. Theory Ser. B, 81(1):58-76, 2001.

[19] V. P. Korzhik and H.-J. Voss. Exponential families of nonisomorphic non-triangular orientable genus embeddings of complete graphs. J. Combin. Theory Ser. B, 86(1):186-211, 2002.

[20] V. P. Korzhik and H.-J. Voss. Exponential families of nonisomorphic nonorientable genus embeddings of complete graphs. J. Combin. Theory Ser. B, 91(4):253-287, 2004.

[21] R. A. Mathon, K.T. Phelps, and A. Rosa. Small Steiner triple systems and their properties. Ars Combin., 15:3-110, 1983.

[22] R. Peltesohn. Eine Lösung der beiden Heffterschen Differenzenprobleme. Compositio Math., 6:251-257, 1939.

[23] G. Ringel. Map color theorem. Springer-Verlag, New York and Berlin, 1974. 